\title{
手机热成像技术在化学实验中的应用研究
}

刘江 $^{\dagger}$, 洪俊华 ${ }^{\dagger}$, 王俐骄, 赵宁东, 徐怀春, 刘俊 ${ }^{*}$

文山学院冶金与环境学院, 云南 文山 663000

摘要: 化学反应常常伴随能量变化, 为获取能量变化信息, 将手机热成像仪 FLIR One Pro 与智能手机组成具有热成像 功能的装置用于化学实验数据采集和处理。对毛细管法测定䒺的熔点、蒸馏法测定乙醇的沸点及浓硫酸与纸的脱水反应 实验中温度动态分布进行可视化观察。得到了不同反应中温度随时间变化的直观图像, 图像颜色变化表征了不同反应过 程的能量变化信息。这种装置能快速、准确、无接触获取体系温度, 能对肉眼不可见的温度变化进行直接观察, 根据颜 色的变化还能显示反应的剧烈程度。

关键词：热成像技术; 温度可视化; 热图像

中图分类号: G64; O6

\section{Application Research of Mobile Phone Thermal Imaging Technology in Chemical Laboratory}

Jiang Liu †, Junhua Hong †, Lijiao Wang, Ningdong Zhao, Huaichun Xu, Jun Liu *

College of Metallurgy and the Environment, Wenshan University, Wenshan 663000, Yunnan Province, China.

\begin{abstract}
Chemical reaction is often associated with the energy changes. The chemical laboratory data of energy changes was recorded on the thermal imaging device made by the phone thermal imaging plug-in FLIR One Pro and smart mobile phone component. The dynamic temperature distribution in the capillary method for the determination of the melting point of naphthalene, the distillation method for the determination of the boiling point of ethanol and the dehydration reaction of concentrated sulfuric acid with paper were visualized. The images of temperature change with time in different reactions were obtained, and the color change of the images represented the energy change information of different reaction processes. This device can quickly, accurately obtain the system temperature without contact, and provides a visualization of the temperature changes invisible to the human eyes.
\end{abstract}

Key Words: Infrared thermography; Visualization of temperature; Infrared thermogram

在自然界中, 物体温度高于绝对零度就会辐射电磁波, 热成像技术能通过采集物体发出的红外 电磁波信号并经处理系统将辐射能量的变化转化为便于肉眼识别直观的温度彩色图谱。热成像技术 具有温度测量范围广、探测准确度高、响应时间短、不会破坏被测温度场等特点 ${ }^{[1]}$, 在民航、电力、 石化、森林防火、医疗等领域应用广泛 ${ }^{[2,3]}$ 。2020年新冠疫情防控中, 红外体温检测仪在车站、口岸、 码头、机场等公众场所对人体体温疑似患者甄别具有重要作用, 是打赢疫情防控战的重要装备, 解 决了传统测温需要人员近距离接触的问题, 实现非接触式快速、精准测温, 减少交叉感染的风险。

收稿：2020-09-14; 录用：2020-11-18; 网络发表：2020-12-06

共同第一作者

“通讯作者, Email: 2382935564@qq.com

基金资助: 文山学院无机化学精品课程建设项目(A2140213); 文山学院校企合作提升大学生创新创业能力项目(JG2018236) 
化学反应都伴随着能量的变化, 这种变化会引起对外辐射电磁波的变化, 热成像技术能捕捉这 种变化并通过数据处理系统转化为对应的温度。2017年美丽科学团队使用高分辨率红外热成像仪拍 摄了 “放热反应” 氢氧化钠溶解、浓硫酸稀释等系列视频并发布在网上供人们观看和下载 ${ }^{[4]}$ 。在传 统的化学实验中, 实验者通常使用水银温度计或皮肤的触觉来感知温度的变化, 普通水银温度计只 能在某特定位置测定该位置的温度, 具有延时、灵敏度低等缺陷, 获得一个温度需要等待几秒甚至 几分钟。

近年来出现了操作简单、便捷的手机红外成像产品, 国内外化学教育教学领域报道了一些在教 学实验中的应用 ${ }^{[5-9]}$, 使用效果好于普通测温工具。段辉等 ${ }^{[10]}$ 采用手机热成像插件巡查实验室, 能消 除潜在的安全隐患; 陈梦麟等 ${ }^{[11]}$ 用手机热成像仪分别拍摄试管和烧杯正确加热与不规范加热造成的 不同结果，这种实时可视化工具可以让实验者看到原本不可见的现象。

本文使用FLIR One Pro手机热成像仪和配套的数据处理系统探究毛细管法测定荎的熔点、蒸馏 法测定乙醇的沸点及浓硫酸与纸脱水反应实验温度分布变化。该种无接触式测温方法, 通过观察热 图像颜色变化表征反应体系温度分布和热量传递过程, 在软件操作界面添加 “测温点” 可对装置同 时进行多点无接触式测温, 配套的数据处理系统将温度变化信号转化为数字信号呈现在手机屏幕。

\section{1 试剂与仪器}

试剂与材料: 䒺、 $95 \%$ 乙醇、浓硫酸(均为分析纯), 铁架台, 硅胶塞, 封口玻璃毛细管, 熔点测 定管, 滤纸, 培养血, 手机三角支架, 蓝牙自拍器。

仪器与设备: 手机红外热成像仪(型号: FLIR ONE PRO 安卓版, 热分辨率: $160 \times 120$, 热像仪 探测器波长范围: $8-14 \mu \mathrm{m}$, 测温范围: $-20-150{ }^{\circ} \mathrm{C}$ 或 $\left.0-400{ }^{\circ} \mathrm{C}\right)$, 智能手机, 201 型蒸馏装置。

\section{2 实验}

\section{1 毛细管法测定芸的熔点}

搭建如图1(a)所示装置, 熔点测定管安装、样品装填等常规程序同教材 ${ }^{[12]}$, 不作赘述。从硅胶塞 上剪下一块略大于熔点测定管口径的硅胶块并用针翟一小孔, 将装有芸的样品管插入小孔并伸入浓 硫酸中, 调节样品高度使其位于支管口附近。开始实验前, 将接有热成像仪的手机用手机三角支架 固定, 启动配套软件操作界面如图1(b) (不同型号手机软件操作界面各部分功能图标分布存在差异, 本文以荣耀AKA-AL10为例), 在软件操作界面上点击右下角 “图像控制” 菜单中的 “调色板” 图标,

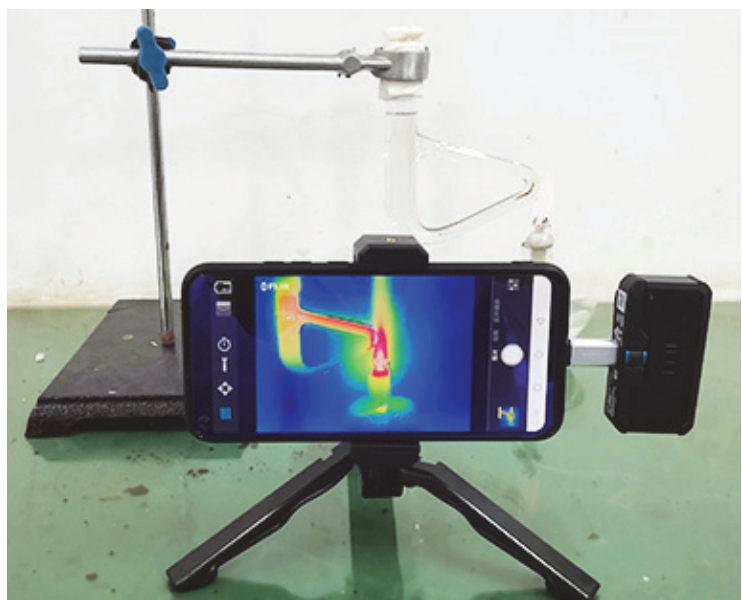

(a)

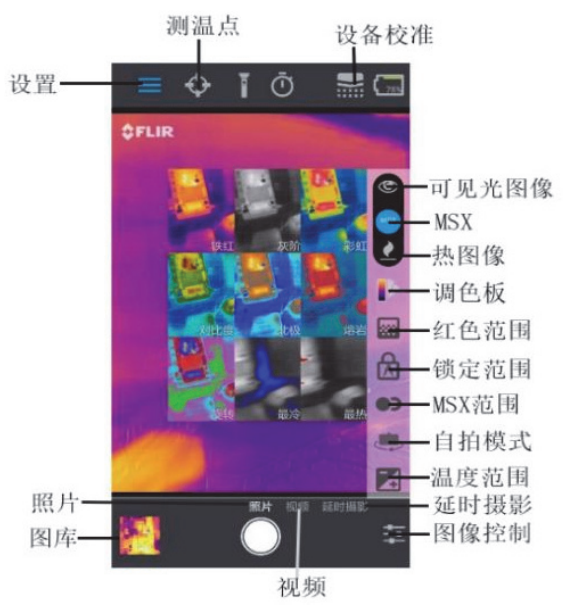

(b)

图1 熔点测定装置(a)与软件操作界面(b) 
更改图像显示方式, 实验者可从 9 种不同的调色板中按需选择, 本文使用的是彩虹图。点击屏幕上方

“测温点” 图标添加测温点并移动至样品所在区域, 调节测温点大小使样品完全位于测温范围内, 可按需添加多个测温点观察同一时刻不同点的温度。下方菜单栏可选择 “照片” 或 “视频” 模式, 点击屏幕底部 “白色圆点” 按钮, 将照片或视频保存至图库中。实验中用蓝牙自拍器辅助拍照或录 制视频记录过程的热图像变化和测温点温度变化，避免因屏幕抖动对实验数据产生影响。

实验结束后点击 “图库” 可查看已保存的图像信息, 选择 “可见图像” 查看自然光状态下的图 像信息, 选择 “热图像” 中 “调色板” 图标可按需再次更换图像显示方式, 图2(a)为热图像(彩虹图), 图2(b)为可见光图像。热图像中蓝色表示低温区域, 红色表示高温区域, 热图像中颜色变化可以直观 反应出整个实验区域温度分布。点击 “测温点” 图标可以按需添加测温点, 移动测温点可获取图像 中任意点温度, 如图2(c)所示。

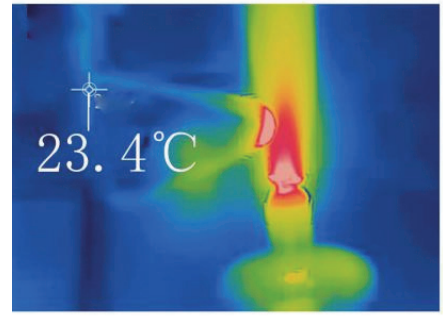

(a)

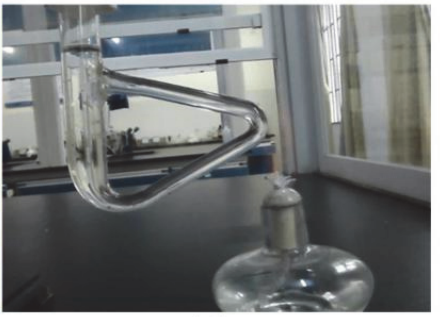

(b)

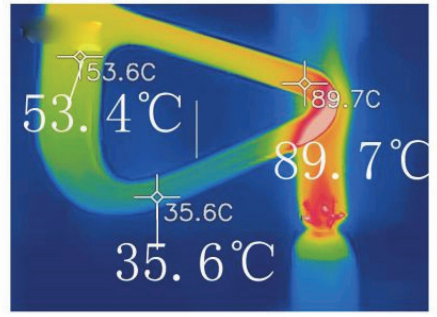

(c)

图2 热图像与可见光图像对比

电子版为彩图

图3为熔点测定管中热量变化过程, 酒精灯靠近熔点测定管加热时热图像对应位置颜色迅速变 红(图3(a)), 热量沿着熔点测定管向加热点上方传递至支管口后逐渐向熔点测定管下方传递, 形成热 对流(图3(b-d))。熔点测定管上部分温度比下部分温度更高, 坚直部分支管口处温度最高。当测温点 温度达到 $79.1{ }^{\circ} \mathrm{C}$ 时样品管内样品出现萎缩塌落、出现小液滴、固液共存、全部熔化等现象, 各阶段 温度清晰可见(图3(e-g)), 样品全部融化后测温点温度继续升高(图3(h))。

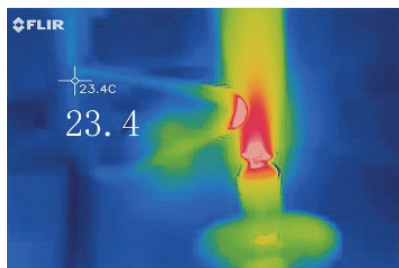

(a)

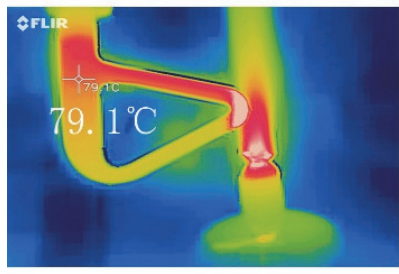

(e)

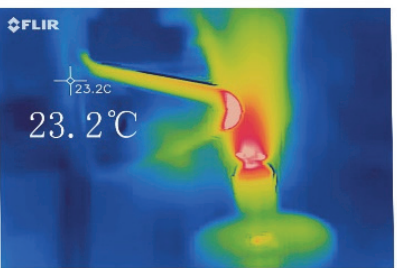

(b)

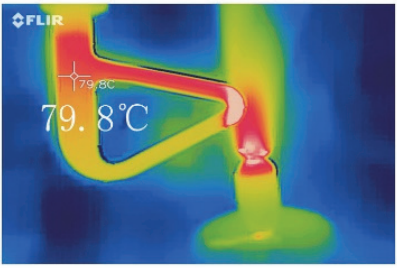

(f)

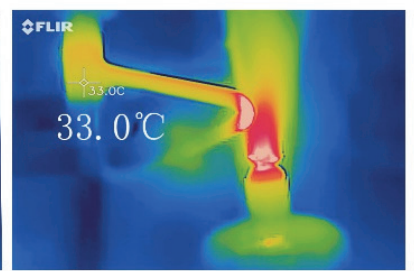

(c)

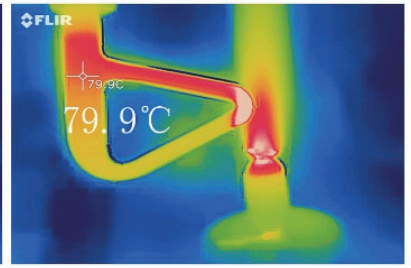

(g)

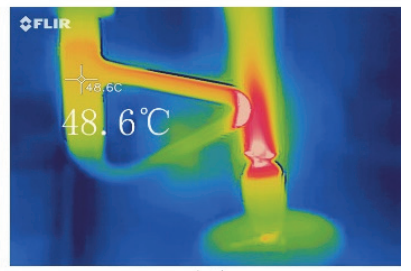

(d)

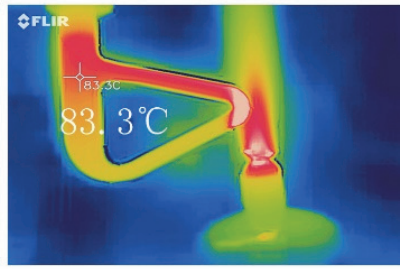

(h)

图3 熔点测定管热图像变化和测温点温度变化 


\section{2 蒸馏法测定乙醇的沸点}

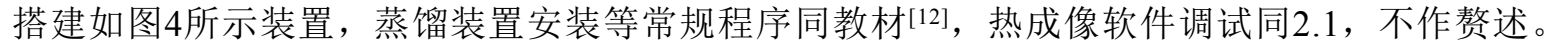
实验环境条件, 室温: $19.7^{\circ} \mathrm{C}$, 大气压: $88.57 \mathrm{kPa}$ 。开始实验前, 添加测温点, 调整支架高度、拍 摄角度及测温点位置使其位于蒸馏头支口, 开启电热装置加热, 通过拍照或录视频记录蒸馏过程支 管口附近的热图像变化和测温点温度变化。

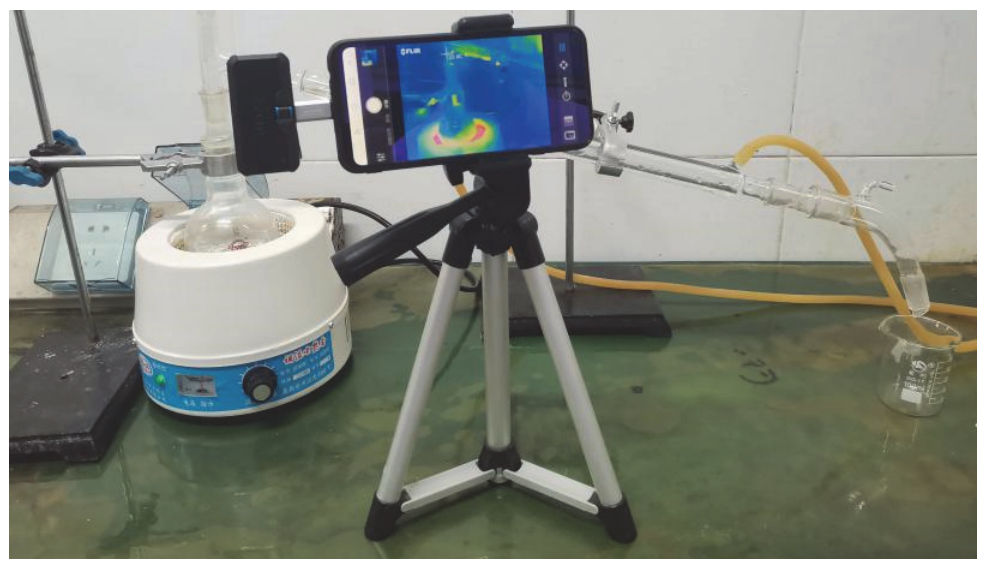

图4 沸点测定装置

图5为蒸馏过程的热图像变化, 开始加热时温度上升缓慢(图5(a-c)), 随后热量从圆底烧瓶向蒸馏 头传递, 蒸馏头支口附近温度逐渐升高(图5d, 5f)。当测温点温度达到 $69.8^{\circ} \mathrm{C}$ 时, 烧瓶中液体开始沸 腾, 接收管口开始有液体滴出, 测温点温度保持在 $69.8^{\circ} \mathrm{C}$ 附近, 接收管口不断有液体滴出(图 $\left.5 \mathrm{~g}, 5 \mathrm{~h}\right)$ 。

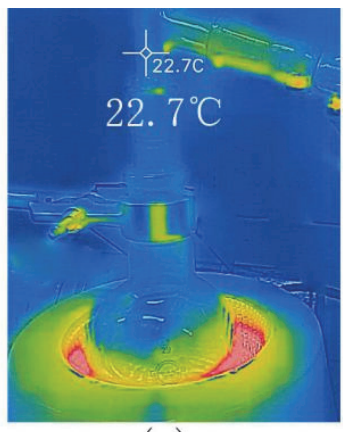

(a)

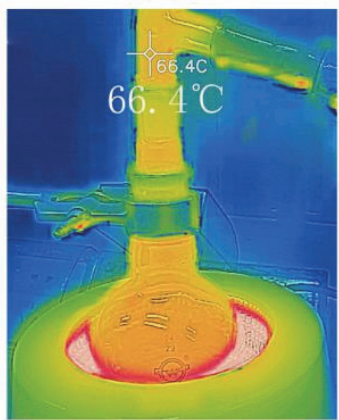

(e)

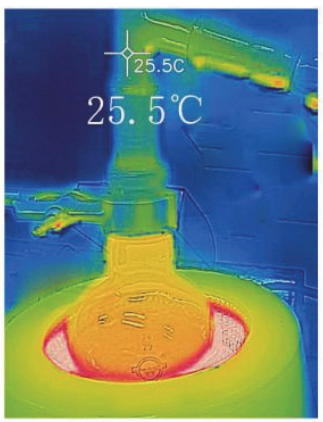

(b)

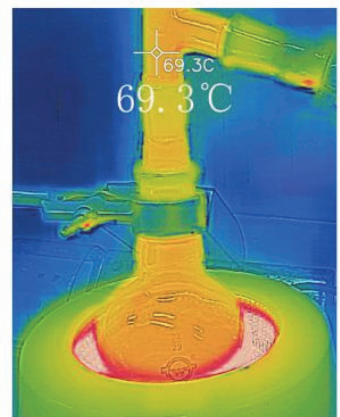

(f)

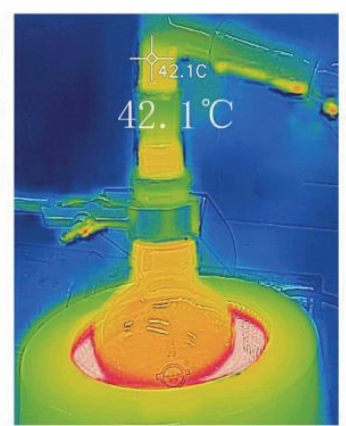

(c)

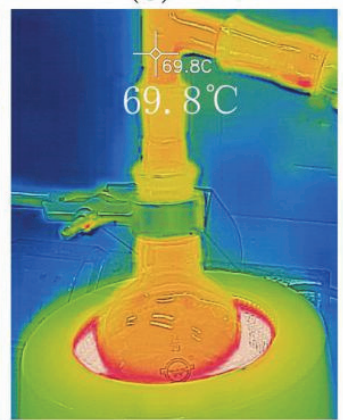

(g)

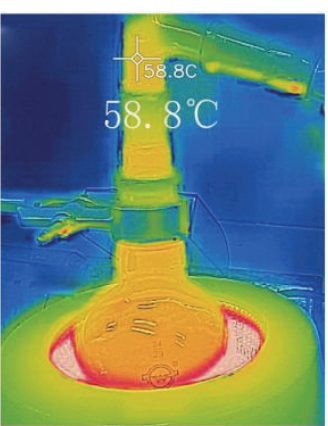

(d)

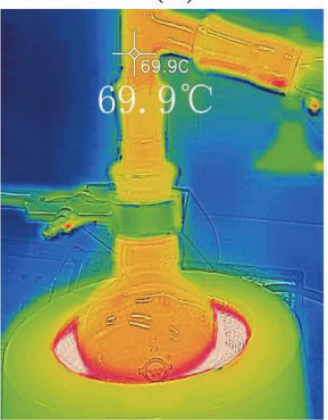

(h)

图5 蒸馏装置热图像变化和测温点温度变化 
通过热成像图像可以看出实验装置中不同位置的温度存在差异, 图像颜色变化可直观反映出热 量在装置中的传递过程及分布情况。普通温度计和一般温度传感器每次只能测定某一点的温度, 无 法同时显示体系各部位的温度分布及动态变化。手机热成像技术可以将被测目标中所有点的温度呈 现在热图像中, 将体系温度变化及热量传递过程转化为图谱中颜色变化, 颜色变化越快说明被测体 系的温度变化越快, 热传递越剧烈。实验结束后可通过观察热图像待装置冷却至合适温度后再拆卸。

\section{3 浓硫酸与纸的脱水反应}

在表面皿上放一张滤纸, 滴加一滴浓硫酸, 用手机热成仪拍摄实验现象。图6为浓硫酸与滤纸脱 水反应过程热图像与可见光对比。

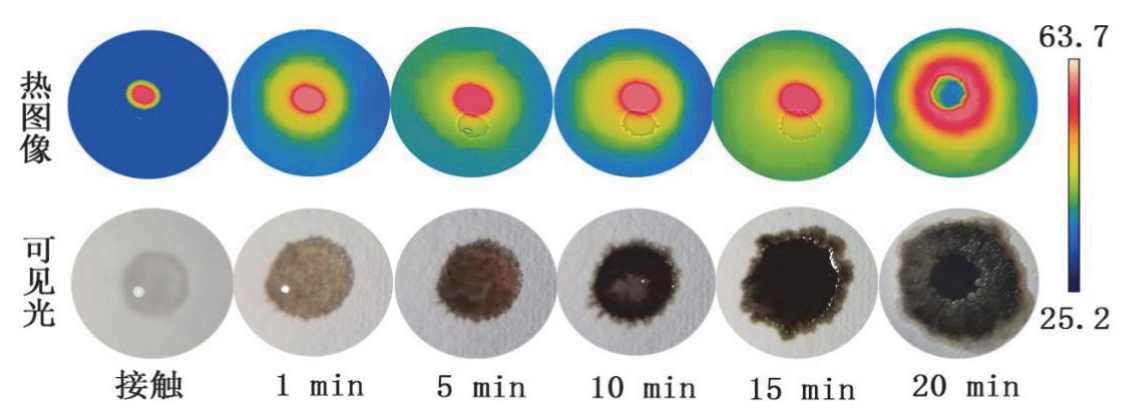

图6 浓硫酸与纸的脱水反过程热图像与可见光图像对比

浓硫酸具有强烈的脱水性, 能将滤纸中的氢、氧元素按水的组成比脱去, 滤纸碳化。浓硫酸滴 到滤纸上, 滤纸表面慢慢变黑, 由接触边缘不断向外延伸, 黑色逐渐加深, 最终在滤纸上腐蚀出一 个孔。通过热图像观察到浓硫酸滴到滤纸表面后颜色迅速变为红色甚至白色, 不同温度呈环状分布。 随着反应的进行中心温度开始逐渐降低, 滤纸被腐蚀穿孔, 该区域颜色变蓝, 温度降至室温, 但四 周温度仍较高。肉眼观察浓硫酸与滤纸的脱水反应过程始终只是单调的黑白两色, 而在热图像中此 过程呈现 “蓝-黄-红” 等多种颜色, 直观表现出脱水放热的特点和滤纸温度分布情况, 在热图像中 添加 “测温点” 能够实时、快速显示出图像任意点温度, 这是一般的目视观察、温度测量或量热法 测量无法做到的。

\section{3 结语}

在化学实验中引入手机热成像技术, 其灵敏度与准确性可以满足一般化学实验教学需求, 在大 学实验教学中具有广阔的应用前景。在有机合成实验中, 用热成像仪能快速、准确、直观地看到反 应体系各部分温度分布, 以此来判断反应物的滴加速度和时间, 有利于提高产率, 如在己二酸的制 备实验中可以通过观察热图像颜色变化来控制已二醇的滴加速率, 有利于提高实验成功率。在微型 实验中, 能精确测定反应的温度, 且不用直接接触, 避免对实验的影响。还可以用来检修实验设备、 电路、管道等, 消除实验室潜在的危险。

在实验教学中依靠手机热成像技术可以把实验过程体系能量变化通过彩色图像直观表达出 来, 能对不可见的热运动和热现象直接观察和分析, 可以加深学生对物理变化、化学变化过程的 理解, 丰富学生的实验体验。

\section{参 考 文 献}

[1] 焦宏光, 高福生. 品牌与标准化, 2016, No. 4, 65.

[2] 张敏, 韩芳, 康键, 孙浩, 郭亮. 红外, 2019, 40 (6), 35. 
[3] 张冀东, 何清湖, 孙涛, 李洪娟, 孙贵香. 中华中医药杂志, 2015, 30 (9), 3202.

[4] 重现化学一放热反应. [2020-06-18]. https://www.envisioningchemistry.cn/

[5] Xu, X.; Wu, M.; Wang, X. J. Chem. Educ. 2019, 96 (11), 2545.

[6] Wong, C. P.; Subramaniam, R. J. Chem. Educ. 2019, 96 (10), 2339.

[7] Xie, C. J. Chem. Educ. 2011, 88 (7), 881.

[8] 凌一洲, 李鳌, 王谦, 任红艳. 化学教育(中英文), 2019, 40 (17), 81 .

[9] 吴梅芬, 王晓岗, 许新华. 实验技术与管理, 2019, 36 (12), 165.

[10] 段辉, 葛晨晨, 许新华. 实验室科学, 2018, 21 (5), 204.

[11] 陈梦麟, 庞春波, 凌一洲, 蔡林轩. 化学教育(中英文), 2020, 41 (15), 67.

[12] 曾和平. 有机化学实验. 第 4版. 北京: 高等教育出版社, 2014: 31-38. 